Denis Le Pesant

Université Paris Ouest Nanterre La Défense \& MoDyCo (CNRS, UMR 7114)

\title{
Essai de classification des prépositions de localisation
}

Nous présentons dans cet article un essai de classification générale des prépositions de localisation prenant en compte le plus grand nombre possible de critères morphologiques, syntaxiques et sémantiques. L'effectif se situe entre 150 et 250 selon les auteurs. Les différences d'estimation peuvent s'expliquer par la disparité des choix théoriques quant à la définition de la catégorie. C'est ainsi que Borillo (1998: 81-82) met en évidence les difficultés de définition de la notion de locution prépositionnelle de localisation. Par exemple, nous nous sommes demandé s'il faut compter ou non comme prépositions de localisation les prépositions de distance (loin, près etc.). Ou bien faut-il ou non, dans les expressions tout en haut, tout à côté etc., compter l'adverbe tout comme partie intégrante de la locution ?

Pour notre part, nous avons relevé jusqu'à présent 209 prépositions de localisation. Dans cet article, qui est une extension de Le Pesant (2011), nous proposons d'abord une définition de la préposition de localisation. Après avoir étudié les principales propriétés morphosyntaxiques de ces propositions, nous présentons notre essai de classification sémantique. Nous présentons aussi, en Annexe, l'état actuel de notre base de données des prépositions de localisation.

\section{Définition des prépositions de localisation}

La préposition de localisation est couramment définie comme étant une préposition qui est la tête d'un Syntagme Prépositionnel commutant avec l'adverbe interrogatif où :

- Nous vivons dans une vieille ferme

- Vous vivez où?

Généralisons cette définition. La préposition de localisation est une préposition qui est la tête d'un Syntagme Prépositionnel commutant avec non seulement l'adverbe interrogatif où, mais aussi le pronom relatif où et les adverbes ici, là, là-bas, quelque part, nulle part, ailleurs, là-dedans, là-dessus etc ${ }^{1}$. Le Syntagme Prépositionnel de localisation figure dans les fonctions syntaxiques suivantes :

- au sein d'une construction attributive :

(2)a Mes livres (sont, resteront) dans une vieille ferme (attribut du sujet)

(2)b Marie a (contemplé, photographié) Paul dans sa baignoire (attribut de l'objet)

- $\quad$ comme ajout dans le Syntagme Verbal :

(2)c Nous nous sommes connus dans cette vieille ferme

- $\quad$ comme ajout de Phrase :

(2)d Dans cette vieille ferme, nous avons passé tant de bons moments

- comme complément locatif des verbes à un seul argument locatif:

(2)e Nous vivons dans une vieille ferme

\footnotetext{
${ }^{1}$ Il est à noter que si le SP de localisation commute avec ces adverbes, l'inverse n'est pas nécessairement vrai, comme le montrent ces exemples :

J'arrive de (Paris, là-bas, où ?) vs *J'arrive d'à Paris

Je suis passé par (une région magnifique, ici, où?) vs *Je suis passé par dans une région magnifique
} 
- comme complément locatif des verbes à plusieurs arguments locatifs (lesquels, tels aller, venir, partir, arriver, sortir, entrer, sont des verbes de déplacement) :

(2)f Nous avons pénétré dans une vieille ferme par une fenêtre laissée ouverte

- $\quad$ en position de Modifieur dans un $\mathrm{SN}$ à tête prédicative :

(2)g le retour d'Ulysse dans sa patrie

Nous empruntons à Z. Harris (cf. Harris 1976: 64-66) le parti de considérer les prépositions de localisation comme des prédicats relationnels à deux arguments ${ }^{2}$. Nous appelons ces deux arguments, respectivement, l'argument entité à localiser et l'argument objet point de repère ${ }^{3}$. L'entité à localiser peut être matérielle (objet concret animé ou inanimé) ou factuelle (état, processus ou événement). On peut donc trouver en position d'argument entité à localiser aussi bien une Phrase qu'un Syntagme Nominal :

$\begin{array}{llll}\text { (3)a } & {[\text { Le tableau La Joconde }]_{\mathrm{SN}}} & \text { est à } & {[\text { Paris }]_{\mathrm{SN}}} \\ (3) \mathrm{b} & {[\text { L'assassinat de Kennedy }]_{\mathrm{SN}}} & \text { a eu lieu à } & {[\text { Dallas }]_{\mathrm{SN}}} \\ (3) \mathrm{c} & {[\text { Kennedy a été assassiné }]_{\mathrm{P}}} & \text { à } & {[\text { Dallas }]_{\mathrm{SN}}}\end{array}$

Dans les exemples (3)a et (3)b, la préposition de localisation à est accompagnée des verbes supports, respectivement, être et avoir lieu. Dans l'exemple (3)c, la préposition est « nue », sans verbe support. Le terme de "préposition de localisation » que nous avons choisi doit s'entendre comme une abréviation de l'expression "préposition de localisation d'une entité par rapport à un point de repère ».

\section{$2 \quad$ Morphosyntaxe des prépositions de localisation}

\subsection{La forme du régime des prépositions de localisation}

Il existe 172 Syntagmes Prépositionnels de forme PREP<localisation $>$ de $S N$ (ex. hors de $\mathrm{N}$, au-dessus de $\mathrm{N}$, à côté de $\mathrm{N}$ ). Parmi ces SP, 4 d'entre eux sont de forme PREP<localisation $>$ de SN et $S N$. Il s'agit de (à égale distance, à équidistance, à l'angle, à l'intersection) de SN et $S N$ :

(4) Le supermarché est à (l'intersection, équidistance) de la rue $A$ et de la rue B

On relève en outre un SP de forme $P R E P<$ localisation $>$ de $S N$, avec une syntaxe de prédicat réciproque :

(5) Le magasin est vis-à-vis de la mairie = Le magasin et la mairie sont vis-à-vis (l'un de l'autre)

Il n'y a qu'un cas de SP de forme $P R E P<$ localisation $>\underline{a} S N$ : il s'agit de face à $N$.

Il y a 4 SP de forme $P R E P<$ localisation $>$ avec $S N$, avec une syntaxe de prédicat réciproque : il s'agit de (côte à côte, dos à dos, face à face, nez à nez) avec $N$ :

$$
A \text { est dos à dos avec } B \text { : A et } B \text { sont dos à dos }
$$

Enfin, il y a 29 SP de forme $P R E P<$ localisation $>\underline{S N}$ (régime « direct »). Il s'agit des prépositions simples (à, dans, devant etc.) qui seront énumérée au $\S 2.2$, plus les locutions en plein dans, en plein sur, loin derrière, loin devant, par-delà, par-derrière, par-dessous, par-dessus, par-devant, très loin derrière, très loin devant.

\footnotetext{
${ }^{2}$ Le fait de voir dans certaines catégories d'adpositions des prédicats à deux arguments correspond aussi (mais avec quelques nuances) à la position de Creissels (2006, tome I: 242), qui est, écrit-il, « motivée par le fait que, mettant à part les emplois régis où leur complément est sémantiquement un argument du mot dont dépend le groupe adpositionnel, on peut reconnaître aux adpositions un signifié relationnel qui en fait potentiellement des prédicats au même titre que les lexèmes verbaux, nominaux ou adjectivaux ».

${ }^{3}$ Les termes utilisés pour désigner ce couple de rôles varient selon les auteurs: lieu/corrélat de lieu (Boons 1985, Guillet \& Leclère 1992), cible/site (Vandeloise 1989), trajector/landmark (Langacker 1987), place/reference object (Jackendoff 1983) etc.
} 
Revenons aux formes PREP<localisation $>($ de, à, avec $S N)$. Il est d'usage d'associer les prépositions de, à, avec aux éléments qui les précèdent, en parlant des prépositions « en face de », «face à » etc. ». Or de, à, avec ne sont que des introducteurs d'argument, voisins des adpositions ou particules casuelles proclitiques portant non sur le nom, mais le SN tout entier. Par exemple, rien ne s'oppose à ce qu'on traite de, dans cet emploi, comme une particule casuelle du génitif (cf. D. Creissels (2006:242-233)) et qu'on dise de prépositions comme hors, autour, auprès, à côté, en bas que ce sont des locutions prépositionnelles à régime génitif.

\subsection{Prépositions simples de localisation}

Nous avons relevé 23 prépositions simples de localisation. Celles qui sont à régime « direct» sont : à, après, avant, chez, contre, dans, derrière, devant, en, entre, par, parmi, sous, sur\#1, sur\#2, sur\#3, vers.

Les prépositions simples de localisation à régime «indirect» sont: (auprès, autour, hors, loin, près) (de $\mathrm{SN})$ et face (à SN)

La préposition par figure ici dans son emploi «non dynamique » (rare en français contemporain) illustré par l'exemple suivant :

(7) Une meute de loups a sévi par les bois et par les champs

La préposition vers figure ici dans l'emploi non directionnel illustré par :

(8) Nous avons fait le plein vers Lyon

Illustrons enfin les trois emplois de sur que nous avons distingués (cf. infra $\S 3)^{4}$ :

$$
\begin{aligned}
& \text { Il y a une assiette sur le vase } \\
& \text { Il y a une mouche sur le plafond } \\
& \text { Il y a des nuages sur Paris }
\end{aligned}
$$

\subsection{Les locutions prépositionnelles de localisation}

Il y a au moins 186 locutions prépositionnelles de localisation.

\subsubsection{Critères du figement, mots à trait d'union et formes de la particule initiale}

Les principaux critères de figement qui se manifestent dans le vocabulaire des locutions prépositionnelles de localisation sont de nature syntaxique : absence de déterminant (à bord, à côté, à droite, en bas, etc.) et impossibilité d'adjoindre un modifieur (* se promener au bord verdoyant d'une rivière, *attendre qq à la porte vitrée d'un bâtiment, *habiter au nord glacial du Canada). En revanche, du point de vue sémantique, on observe que le sens de ces locutions est souvent parfaitement calculable, ce qui nous permettra (cf. Partie 3) de dériver dans une large mesure la classification sémantique des locutions prépositionnelles de celle de ces noms. Cette contradiction entre le figement syntaxique et le caractère compositionnel du sens peut s'expliquer par le processus de grammaticalisation (cf. infra § 2.3.4) qui a donné naissance à la majorité de ces prépositions.

Parmi les locutions prépositionnelles de localisation, il y a 10 mots à trait d'union (au-dedans, au-dehors, au-delà, au-dessous, au-dessus) pour lesquels il existe les variantes graphiques au dedans, au dehors, au delà, au dessous, au dessus. Les autres mots à trait d'union sont par-delà, par-derrière, par-dessous, pardessus, par-devant. Ces prépositions figurent dans leur emploi «non dynamique » (rare en français contemporain) illustré par :

$$
\begin{aligned}
& \text { Le ciel est par-dessus les toits } \\
& \text { La vente a été conclue par-devant mon notaire de famille }
\end{aligned}
$$

\footnotetext{
${ }^{4}$ A ces trois emplois, il faudra sans doute bientôt ajouter celui-ci, récent, illustré par :

Trois jours par semaine, je suis sur Lille
} 
Examinons la forme de la particule initiale : 109 locutions commencent par la particule à (à deux pas, à la cime etc.) ; 24 locutions commencent par la particule en (en amont, en deçà, en arrière etc.) ; 15 locutions commencent par la particule de (de ce côté-ci, de bas en haut etc.).

\subsubsection{Les variantes intensives}

Un certain nombre de prépositions de localisation comportent une variante intensive de forme adverbiale ou adjectivale. Les formes adverbiales sont tout (tout à côté, tout à l'entour, tout au bout, tout au fond, tout au long, tout au milieu, tout autour, tout contre, tout du long, tout en bas, tout en haut, tout le long, tout près) ; loin (loin derrière, loin devant) ; très (très loin, très près, très loin derrière, très loin devant etc.) ; le plus (au plus profond).

Les formes «adjectivales" sont : plein (en plein centre, en plein dans, en plein milieu, en plein sur); beau (au beau milieu); fin (au fin fond); respectueuse (à distance respectueuse, à une distance respectueuse).

2.3.3 Les classes de noms figurant au sein des locutions prépositionnelles de localisation et leur nature

Nombre de locutions de localisation comportent un nom susceptible de figurer en emploi libre dans la construction de forme :

(11)a $\quad$ Le $N$ de $N<$ concret $>$

(11)b L'intérieur de cette maison est délabré et l'extérieur est recouvert de lierre

Il y a une étiquette sur le dessus de ce coffret; les alentours de la ville sont charmants

La frontière du Maroc est fermée ; l'arrière de ma voiture est cabossé

Parmi ces noms figurent principalement des noms de portions (ex. le haut, le bas, l'avant, l'arrière, le côté, le bout etc.) et des noms de termes. Cette dernière catégorie se subdivise en deux classes : les noms de limites (ex. le dessus, le dessous, le devant, le derrière, le bord etc.) et les noms de terme intermédiaire ou milieu (le milieu, le centre). Les noms de portions et de limites peuvent être «polarisés » selon les axes vertical, frontal, latéral ou selon les axes des points cardinaux.

Parmi ces noms figurent aussi des noms originellement de partie du corps (ex. le cour, le pied, la tête, les yeux, la main etc.) et de parties de bâtiment, paysage, pays etc. (ex. la porte, la cime, la lisière, la frontière etc.). Ces catégories peuvent être souvent rapprochées des noms de portions et de termes : par exemple lisière est un nom de limite et cour est un nom de milieu.

Quelle est la nature de ces noms ? S'agit-il de méronymes ? Il est difficile de le soutenir car ils sont bien plus syncatégorématiques (cf. G. Kleiber (2001: 283) que les méronymes. En effet, contrairement aux méronymes, ils sont presque tous inaptes à avoir un référent autonome :

(12)a *J'ai photographié (un bord, un haut, un centre, un dessus, un côté, un bout)

vs

(12)b J'ai photographié (une main, un toit, une roue, une portière, une branche, des racines)

Les noms qui figurent dans l'exemple (12)a peuvent être rapprochés de noms comme portion, morceau, moitié, totalité, bout : les uns et les autres sont tout à fait syncatégorématiques et sont par conséquent dans l'impossibilité d'être analysés comme étant la tête du SN, dans des expressions telles que :

(13)a le bord du trottoir, le haut du buffet, le centre du jardin, le bout du couloir

cf.

(13)b (une portion, un morceau) de tarte ; la moitié de la population ; un bout de pain

Tous ces mots sont morphologiquement des noms ; mais du point de vue syntactico-sémantique, ce sont des déterminants. Nous qualifions de déterminants locatifs les noms syncatégorématiques haut, bas, devant, 
extrémité, côtés, bout etc. En effet, ils désignent l'effet d'une partition sur l'extension spatiale d'un objet concret $^{5}$.

2.3.4 Processus de grammaticalisation ayant donné lieu à la majorité des locutions prépositionnelles de localisation

Dans l'histoire de la langue, une préposition simple à, en ou de en est venue à s'associer, dans le cadre d'un processus de grammaticalisation (cf. Hopper \& Traugott 1993), au premier élément d'un syntagme de forme le $N$ de-SN, de façon à former avec lui une locution de forme $(\grave{\boldsymbol{a}}, \boldsymbol{e n}, \boldsymbol{d e})(\boldsymbol{l e}, \boldsymbol{0}) \boldsymbol{N}$. On passe ainsi de (14)a à (14)b :

(14)a Syntaxe «primitive»

\begin{tabular}{|l|c|l|l|}
\hline Argument entité à localiser & Prépositions simples & \multicolumn{2}{|c|}{ Argument point de repère } \\
\cline { 3 - 4 } & & DET locatifs & de SN \\
\hline Pierre & est à & l'intérieur & de sa chambre \\
\hline On a mangé & en & haut & de la colline \\
\hline L'incendie fait rage & $\grave{a}$ & le bord & du fleuve \\
\hline
\end{tabular}

(14)b Syntaxe « accomplie »

\begin{tabular}{|l|c|l|}
\hline \multicolumn{1}{|c|}{ Argument entité à localiser } & Locutions prépositionnelles & Argument point de repère \\
\hline Pierre & est à l'intérieur & de sa chambre \\
\hline On a mangé & en haut & de la colline \\
\hline L'incendie fait rage & au bord & du fleuve \\
\hline
\end{tabular}

\subsubsection{L'emploi absolu des locutions prépositionnelles de localisation}

Le régime de la majorité des prépositions (149 sur 205), tant simples que locutionnelles, peut faire l'objet d'une ellipse à condition que l'élément effacé soit récupérable dans le contexte discursif (ellipse anaphorique) ou par prise en compte du contexte d'énonciation (ellipse déictique) :

Tu vois l'église? J'habite (à côté, en face) (de l'église, 0)

Il s'agit d'un cas particulier d'anaphore associative (cf. Le Pesant (1996)), cela près qu'on n'est pas en présence d'une relation méronyme/holonyme.

Les principales prépositions locatives qui refusent l'ellipse de leur régime (57 sur 205) sont notamment : les prépositions simples dans, sous, sur, hors (lesquelles ont remplacé les anciennes prépositions dedans, dessous, dessus et dehors qui l'acceptaient et qui subsistent en français moderne en tant qu'adverbes anaphoriques ou déictiques) ; les prépositions simples à, contre, en, entre, parmi, vers, face, auprès ; des locutions formées à partir de noms de parties du corps (à la barbe, à la droite, à la gauche, au cour, au sein, aux pieds, aux côtés, au pied, sous le nez, sous les yeux). D’autres prépositions sont concernées, telles au pourtour, par-delà, au long, le long, à la hauteur, au contact, au niveau, au ras, au voisinage, aux abords etc ${ }^{6}$.

\footnotetext{
${ }^{5}$ On peut aussi rapprocher les déterminants locatifs des déterminants de phase (le début, le commencement, la fin etc.) qui signifient quant eux une partition sur l'extension temporelle d'un événement.

${ }^{6}$ Quelques prépositions comportant un nom de partie du corps et sélectionnant un nom d'humain peuvent incorporer un déterminant déictique possessif: (à, sur) ma (droite, gauche) ; à mes (pieds, côtés), sous (mon nez, mes yeux). Ce fait est conforme aux conditions générales pesant sur l'anaphore associative quand un nom de partie du corps est en cause :

une église ... le clocher ... ; un homme ... * la tête ... ; un homme ... sa tête ...
} 
3.1 Présentation générale de la méthode

Nous postulons que la sémantique des locutions prépositionnelles est en règle générale héritée de celle des noms déterminants locatifs qui viennent d'être évoqués, et qui subsistent dans l'environnement figé des locutions prépositionnelles tout en conservant leur valeur sémantique (cf. supra § 2.3.1). Plus précisément, nous postulons que, dans les diverses sortes de relations de localisation interne ou externe, ce n'est pas l'objet point de repère en tant que tel qui est mis en rapport avec l'entité à localiser, mais seulement une de ses portions ou un de ses termes (limite ou milieu), lesquels remplissent la fonction de «médiateurs de la relation de localisation ». Soient les exemples :

(16)a Nous étions à l'extérieur du cinéma

(16)b Les autres étaient déjà à l'intérieur du cinéma

(16)c Il y a un pommier au centre du jardin

La phrase (16)a signifie une relation de localisation externe entre l'entité à localiser («nous ») et la limite de l'objet à localiser qui s'appelle «l'extérieur du cinéma », c'est-à-dire la limite externe du cinéma. Cette analyse est fondée sur le constat que le nom extérieur (comme d'ailleurs le nom dehors) désigne d'abord la limite d'un objet (par exemple dans l'extérieur de la maison est fissuré).

La phrase (16)b signifie une relation de localisation interne entre l'entité à localiser «les autres » et les limites de l'objet à localiser. Cette analyse est fondée sur le constat de la relation linguistique existant entre les structures suivantes :

$$
\begin{aligned}
& \hat{e} \text { tre à l'intérieur de qqch } \\
= & \hat{e} \text { tre entre les limites de qqch } \\
= & \hat{e} \text { tre à l'intérieur des limites de qqch }
\end{aligned}
$$

Dans la phrase (16)c, l'entité à localiser « pommier» est mise en relation avec le terme intermédiaire (le milieu) « centre », dans un rapport qu'on ne peut qualifier autrement que de localisation externe : c'est un rapport de localisation externe par rapport au centre. Ce rapport implique une relation de localisation interne par rapport à la limite externe ; le statut de préposition de «localisation interne » généralement attribué aux prépositions au centre et au milieu n'est donc qu'implicite.

\subsection{Définition des relations de localisation interne et de localisation externe}

A. Borillo (1998) et ses disciples, tels Aurnague (1991), Laur (1991), Vieu (1991), Sablayrolles (1995) ont popularisé les notions antithétiques de localisation interne et de localisation externe. Pour notre part, nous définissons les relations de localisation interne et de localisation externe en termes de directions centripète vs centrifuge par rapport aux limites de l'objet point de repère. Est localisée à l'intérieur toute entité située entre une limite de l'objet point de repère et son centre, ou entre ses limites; d'autre part, est localisée à l'extérieur, à partir de la limite de l'objet point de repère, toute entité située dans la direction opposée à celle du centre.

D'autre part, la relation de localisation peut se faire non seulement avec les limites de l'objet point de repère, mais aussi avec celles d'une de ses portions (désignées par des noms tels que le haut, le bas, le côté, l'avant, l'arrière). Soit par exemple la phrase :

\section{Il y a une photo en haut de l'armoire}

Dans un de ses sens, cette phrase peut être paraphrasée de la façon suivante :

(17)b «Il y a une photo à l'intérieur de la portion haute de l'armoire »

La relation de localisation interne s'établit alors, dans cet emploi particulier, dans le champ limité de la portion haute. 
La localisation externe peut être avec ou sans contact. Par exemple, il existe un emploi de localisation externe sans contact de la préposition sur (ex. Il y a des nuages sur la ville); dans un autre emploi, la localisation est de contact avec une limite supérieure (ex. Il y a des livres sur le piano). Dans bien des cas, il existe une incertitude sur la présence ou l'absence de contact; c'est ce qui se passe avec la sémantique de prépositions telles que au-dessus, au nord, à côté.

3.4 Les équivoques sur le type de localisation

Nous venons d'évoquer un cas d'équivoque sur le type de localisation. En voici quelques autres. Reprenons l'exemple de la phrase (17)a. Elle a deux autres interprétations possibles :

(17)c «Il y a une photo sur un côté, en haut de l'armoire »

(17)d «Il y a une photo sur le dessus de l'armoire »

Prenons un dernier exemple d'équivoque, dans la phrase :

(18)a Je t'attends en bas de ton immeuble

Elle a les deux interprétations suivantes :

(18)b « Je t'attends en bas de ton immeuble, à l'intérieur »

(18)c «Je t'attends en bas de ton immeuble, à l'extérieur»

3.5 Les principales catégories de prépositions de localisation

Les principales catégories de prépositions de localisation sont :

- les prépositions de localisation interne par rapport à une limite ou une portion

- les prépositions de localisation interne ou externe avec contact par rapport à une limite ou une portion

- les prépositions de localisation externe avec ou sans contact par rapport à une limite ou une portion

- les prépositions de localisation externe avec contact par rapport à des limites (y compris les surfaces)

- les prépositions de localisation externe sans contact par rapport à des limites

- les prépositions de localisation externe avec contact par rapport au terme intermédiaire (le milieu)

3.5.1 Les prépositions de localisation interne par rapport à une limite ou une portion

Sont prototypiques de la catégorie les prépositions de localisation interne par rapport à la limite totale d'un objet à trois dimensions : dans, à l'intérieur, au-dedans etc. Les principales autres catégories sont les prépositions de localisation par rapport par un couple de limites latérales (à travers, au travers ...) et par rapport à un couple de portions (de haut en bas, de bas en haut ...). Ces deux dernières catégories se caractérisent par leur effet holistique (cf. Anderson 1971).

3.5.2 Les prépositions de localisation interne et externe avec contact par rapport à une limite ou une portion

Prenons l'exemple de la préposition à dans deux de ses principaux emplois (sélection des noms de villes et des descriptions définies); elle signifie de façon équivoque la localisation interne et la localisation externe avec contact : on est à Paris aussi bien quand on est arrivé aux portes de Paris que quand on est déjà au centre-ville. On relève notamment, dans la même catégorie, les prépositions de localisation par rapport à : des limites latérales (au bord, en bordure, du côté droit ...), des portions courbes de voies (au tournant, au détour), à des portions extrêmes (au bout, à l'extrémité, à l'autre bout ...), des portions antérieures et postérieures (à l'avant, à l'arrière, en tête ...), des portions inférieures et supérieures (au bas, en haut, tout en haut, au sommet ...).

3.5.3 Les prépositions de localisation externe avec ou sans contact par rapport à une limite ou une portion

On relève notamment, dans cette catégorie, les prépositions de localisation par rapport à : une limite totale (hors, à l'extérieur, au-dehors ...) ; des limites circulaires (autour, à la périphérie, au pourtour ...) ; des limites frontales antérieures et postérieures (devant, avant, derrière, après, par-delà ...); des limites 
latérales droite et gauche (à droite, à main droite, à tribord, à gauche ...); une limite longue (le long, le long ...) ; un objet ou localité sur un cours d'eau (en amont, en aval); une limite inférieure et supérieure (au-dessus, en dessous ...); à une portion cardinale (au nord ...); à une portion latérale (à côté, de chaque côté) etc.

3.5.4 Les prépositions de localisation externe avec contact par rapport à des limites ou surfaces

Il existe un emploi de la préposition sur comme prédicat de localisation externe avec contact par rapport à une limite verticale supérieure, ainsi que quelques prépositions de localisation externe avec contact par rapport à des limites de type « ouvertures » (à la porte, à l'entrée, à la sortie ...). Dans un autre de ses trois emplois principaux, la préposition sur est un prédicat de localisation externe par rapport une surface (ex. une mouche se promène sur le plafond). Voici d'autres prépositions appartenant à la même catégorie : au ras, à la surface, au revers, à l'envers, au dos, au verso.

\subsubsection{Les prépositions de localisation externe sans contact par rapport à des limites}

La plupart des prépositions de localisation externe sans contact par rapport à des limites sont des prédicats de distance grande ou petite : loin, à distance respectueuse, près, à proximité, aux abords, vers ... Quelques prépositions signifient une relation externe sans contact par rapport à un couple de limites : entre, à égale distance, à équidistance.

3.5.6 Les prépositions de localisation externe avec contact par rapport au terme intermédiaire (le milieu)

Les principales prépositions de localisation externe avec contact par rapport à un terme intermédiaire (milieu) sont d'une part au milieu, au beau milieu, au centre, au cour, au sein etc., d'autre part à mihauteur, à mi-largeur, à mi-longueur, à mi-côte, à mi-pente, à mi-profondeur.

\section{Conclusion}

D'autres critères permettent d'affiner la classification. Ce sont notamment d'une part les critères d'origine de la polarité : déictique ou essentielle; par exemple, la polarité frontale antérieure peut être essentielle quand elle est propre aux noms désignant un objet pourvu d'une «façade »; elle est déictique quand elle dépend du point du point de vue du locuteur. Un autre critère de classification est celui des propriétés de restriction lexicale sur l'argument point de repère : humain, objet allongé, étendue d'eau, bateau, forêt etc., dont n'avons fait que l'esquisse. D’une façon générale, beaucoup de champs figurant dans la base de données que nous présentons en Annexe ont besoin d'être corrigés et complétés. Du reste, chacune des 206 prépositions de localisation mérite de donner lieu à une notice particulière.

\section{Références}

Borillo, A. (1998). L'espace et son expression en français. Paris : Ophrys.

Creissels, D. (2006). Syntaxe générale, une introduction typologique. Paris : Hermès-Lavoisier.

Guillet, A. \& Leclère, C. (1992). La structure des phrases simples en français : constructions locatives transitives. Genève : Droz.

Harris, Z. (1976). Notes du cours de syntaxe. Paris : Le Seuil.

Hopper, P. J. \& E. Traugott. (1993). Grammaticalization. Cambridge University Press.

Jackendoff, R. (1983). Semantics and Cognition. Cambridge Massachussets : MIT Press.

Kleiber, G. (2001). L'anaphore associative. Paris : PUF.

Langacker, R.W. (1987). Foundations of Cognitive Grammar, vol. I. Theoretical Prerequisites. Stanford : Stanford University Press.

Laur, D. (1991). Sémantique du déplacement et de la localisation en français: une étude des verbes, des prépositions et de leurs relations dans la phrase simple. Toulouse : Thèse Université Paul Sabatier. 
Le Pesant, D. (1996). « Anaphores associatives et classes d'objets », in Lingvisticae Investigationes XX,1:87-116. Amsterdam : John Benjamins Publishing Company.

Le Pesant, D. (2011). «Problèmes de morphologie, de syntaxe et de classification sémantique dans le domaine des prépositions locatives ", in Au commencement était le verbe. Syntaxe, Sémantique et Cognition. Mélanges en l'honneur du Professeur Jacques François (dir. F. Neveu, P. Blumenthal et N. Le Querler). Bern, Berlin : Peter Lang.

Sablayrolles, P. (1995). Sémantique formelle de l'expression du mouvement. Toulouse : Thèse Université Toulouse III.

Vandeloise, C. (1986): L'espace en français. Paris : Le Seuil.

Vieu, L. (1991). Sémantique des relations spatiales et inférences spatio-temporelles: une contribution à l'étude des structures formelles de l'espace. Toulouse : Thèse Université Paul Sabatier. 
ANNEXE

Base de données des Prépositions de Localisation (Version 1)

\begin{tabular}{|c|c|c|c|c|c|c|c|c|c|c|}
\hline PREP. LOCATIVES & Var.graph. & $\begin{array}{l}\text { simple vs } \\
\text { locution }\end{array}$ & Régime & $\begin{array}{l}\text { Emploi } \\
\text { absolu }\end{array}$ & Anaphores & $\begin{array}{l}\text { Médiateur de la } \\
\text { localisation }\end{array}$ & LI/LE & $\begin{array}{l}\text { Polarité, } \\
\text { etc. }\end{array}$ & Grandeurs etc. & $\begin{array}{l}\text { Restrict. de sélect. } \\
\text { lexicale }\end{array}$ \\
\hline à & & simple & $\mathrm{N}$ & non & & lim. totale & LI, LE + contact & & & $\begin{array}{l}\text { N propre toponyme, } \\
\text { Descr. déf. }\end{array}$ \\
\hline à bâbord & & à $\mathrm{N}$ & de $N$ & oui & & lim. latér. gauche & LE +/-contact & essent. & & bateau \\
\hline à bord & & à $\mathrm{N}$ & de $\mathrm{N}$ & oui & & lim. totale & LI & & & $\begin{array}{l}\text { bateau, moyen de transp. } \\
\text { coll. }\end{array}$ \\
\hline à card Nunit. & & à card $\mathrm{N}$ & de N & oui & & lim. totale & LE -contact & & dist. exacte & \\
\hline à côté & & à $\mathrm{N}$ & de $N$ & oui & & portion latér. & LE +/-contact & & dist. près & \\
\hline à deux pas & & à deux N & de $N$ & oui & & lim. totale & LE -contact & & dist. près & \\
\hline à distance & & à $\mathrm{N}$ & de $N$ & oui & & lim. totale & LE -contact & & dist. Ioin & \\
\hline $\begin{array}{l}\text { à distance } \\
\text { respectueuse }\end{array}$ & & $\begin{array}{l}\grave{a} \mathrm{~N} \\
\text { respectueuse }\end{array}$ & de $N$ & oui & & lim. totale & LE -contact & & dist. Ioin & \\
\hline à droite & & à $\mathrm{N}$ & de N & oui & & lim. latér. droite & LE +/-contact & déict. & & \\
\hline à égale distance & & à ADJ N & de $\mathrm{N}$ et $\mathrm{N}$ & non & & lim. totale : 2 & LE -contact & & dist. équidist. & \\
\hline à équidistance & & à $\mathrm{N}$ & de $\mathrm{N}$ et $\mathrm{N}$ & non & & lim. totale : 2 & LE -contact & & dist. équidist. & \\
\hline à gauche & & à N & de $N$ & oui & & lim. latér. gauche & LE +/-contact & déict. & & \\
\hline à hauteur & & à $N$ & de $N$ & oui & & lim. totale & LE +/-contact & & alignement & \\
\hline à la barbe & & à la N & de $N$ & non & DET poss. & lim. front. antér. & LE -contact & & dist. près & HUM \\
\hline à la cime & & à la N & de $N$ & non & & portion vertic. supér. & LI, LE + contact & & & bât, végét \\
\hline à la droite & & à la N & de $N$ & non & DET poss. & lim. latér. droite HUM & LE +/-contact & essent. & & HUM \\
\hline à la frontière & & à la N & de $N$ & oui & & lim. totale & LI, LE + contact & & & Etat \\
\hline à la gauche & & à la N & de $N$ & non & DET poss. & lim. latér. gauche HUM & LE +/-contact & essent. & & HUM \\
\hline à la hauteur & & à la $N$ & de $N$ & non & & lim. totale & LE +/-contact & & alignement & \\
\hline à la lisière & & à la N & de $N$ & oui & & lim. totale & LI, LE + contact & & & bois, forêt \\
\hline à la périphérie & & à la N & de N & oui & & lim. circulaire & LE +/-contact & & & \\
\hline à la porte & & à la N & de N & oui & & lim. ouverture & LE +contact & & & \\
\hline à la sortie & & à la N & de $N$ & oui & & lim. ouverture & LE +contact & déict. & & \\
\hline à la surface & & à la N & de N & oui & & surface & LE + contact & & & \\
\hline à l'angle & & à le $\mathrm{N}$ & de $\mathrm{N}$ et $\mathrm{N}$ & non & & lim. angulaire & LI, LE + contact & & & 2 objets allongés \\
\hline à l'aplomb & & à le N & de $N$ & oui & & lim. totale & LE +/-contact & & aplomb & \\
\hline à l'arrière & & à le N & de $N$ & oui & & portion front. postér. & LI, LE + contact & & & \\
\hline à l'autre bout & & à l'autre $\mathrm{N}$ & de N & oui & & portion extrême & LI, LE + contact & déict. & & objet long \\
\hline à l'autre extrémité & & à l'autre N & de $N$ & oui & & portion extrême & LI, LE + contact & déict. & & objet long \\
\hline
\end{tabular}




\begin{tabular}{|c|c|c|c|c|c|c|c|c|c|}
\hline à l'avant & & à le N & de N & oui & portion front. antér. & LI, LE + contact & & & \\
\hline à l'écart & & à le N & de N & oui & lim. totale & LE -contact & & dist. près & \\
\hline à l'entour & alentour & à le N & de N & oui & lim. circulaire & LE +/-contact & & & \\
\hline à l'entrée & & à la N & de N & oui & lim. ouverture & LE +contact & déict. & & \\
\hline à l'envers & & à le N & de N & oui & surface verso & LE +contact & & & \\
\hline à l'est & & à le N & de N & oui & portion card. est & LE +/-contact & & & \\
\hline à l'extérieur & & à le N & de N & oui & lim. totale & LE +/-contact & & & \\
\hline à l'extrémité & & à la N & de N & oui & portion extrême & LI, LE + contact & & & objet long \\
\hline $\begin{array}{l}\text { à l'extrémité } \\
\text { ADJpolar. }\end{array}$ & & à la N & de N & oui & portion extrême & LI, LE + contact & & & objet long \\
\hline à l'intérieur & & à le $\mathrm{N}$ & de N & oui & lim. totale & $\mathrm{LI}$ & & & \\
\hline à l'intersection & & à la N & $\operatorname{de} \mathrm{N}$ et $\mathrm{N}$ & oui & lim. angulaire & LI, LE + contact & & & 2 objets allongés \\
\hline à l'orée & & à la N & de N & non & lim. totale & LI, LE + contact & & & bois, forêt \\
\hline à l'ouest & & à le N & de N & oui & portion card. ouest & LE +/-contact & & & \\
\hline à main droite & & à N ADJ & de N & oui & lim. latér. droite & LE +/-contact & déict. & & HUM \\
\hline à main gauche & & à N ADJ & de N & oui & lim. latér. gauche & LE +/-contact & déict. & & HUM \\
\hline à mi-côte & & à mi-N & de N & oui & milieu & LE +contact & & dimens. pente & \\
\hline à mi-hauteur & & à mi-N & de N & oui & milieu & LE + contact & & dimens. hauteur & \\
\hline à mi-largeur & & à mi-N & de N & oui & milieu & LE + contact & & dimens. largeur & \\
\hline à mi-longueur & & à mi-N & de N & oui & milieu & LE + contact & & dimens. Iongueur & \\
\hline à mi-pente & & à mi-N & de N & oui & milieu & LE + contact & & dimens. pente & \\
\hline à mi-profondeur & & à mi-N & de N & oui & milieu & LE +contact & & $\begin{array}{l}\text { dimens. } \\
\text { profondeur }\end{array}$ & étendue d'eau \\
\hline à niveau & & à N & de N & oui & lim. totale & LE +/-contact & & alignement & \\
\hline à portée & & à N & $\operatorname{deN}$ & oui & lim. totale & LE -contact & & dist. près & objet, HUM \\
\hline à proximité & & à $N$ & de N & oui & lim. totale & LE -contact & & dist. près & \\
\hline à quelque distance & & à quelque $\mathrm{N}$ & de N & oui & lim. totale & LE -contact & & dist. près & \\
\hline à quelques Nunit & & à quelques $\mathrm{N}$ & de N & oui & lim. totale & LE -contact & & dist. exacte & \\
\hline à ras & & $\overline{\mathrm{àN}}$ & de N & oui & surface & LE + contact & & alignement & \\
\hline à travers & & à N & $\bar{N}$ & oui & lim. latér. : 2 & $\mathrm{LI}$ & holist. & & objet allongé \\
\hline à tribord & & $\overline{\mathrm{àN}}$ & de N & oui & lim. latér. droite & LE +/-contact & essent. & & bateau \\
\hline à un bout & & à un $\mathrm{N}$ & de N & oui & portion extrême & LI, LE + contact & & & objet long \\
\hline $\begin{array}{l}\text { à une distance de } \\
\text { card Nunit }\end{array}$ & & $\begin{array}{l}\text { à une } \mathrm{N} \text { de card } \\
\text { Nunit }\end{array}$ & de N & oui & lim. totale & LE -contact & & dist. exacte & \\
\hline $\begin{array}{l}\text { à une distance } \\
\text { respectueuse }\end{array}$ & & $\begin{array}{l}\text { à une } \mathrm{N} \\
\text { respectueuse }\end{array}$ & de N & oui & lim. totale & LE -contact & & dist. loin & \\
\hline à une extrémité & & à une $\mathrm{N}$ & de N & oui & portion extrême & LI, LE + contact & & & objet long \\
\hline
\end{tabular}




\begin{tabular}{|c|c|c|c|c|c|c|c|c|c|}
\hline après & simple & $\mathrm{N}$ & oui & & lim. front. postér. & LE +/-contact & déict./essent. & & \\
\hline au bas & au N & de $N$ & non & & portion vertic. infér. & LI, LE + contact & & & \\
\hline au beau milieu & au beau $\mathrm{N}$ & de $N$ & oui & & milieu & LE + contact & LI impl. & & objet 1D/2D/3D, collection \\
\hline au bord & au N & de $N$ & oui & & lim. latér. & LI, LE + contact & & & $\begin{array}{l}\text { surf. longue, circ./creuse, } \\
\text { eau }\end{array}$ \\
\hline au bout & au N & de N & oui & & portion extrême & LI, LE +contact & déict. & & objet long \\
\hline au centre & au N & de $N$ & oui & & milieu & LE + contact & LI impl. & & objet 2D/3D \\
\hline au centre-ville & au N & de $N$ & oui & & milieu & LE + contact & Ll impl. & & localité \\
\hline au cœur & au N & de $N$ & non & & milieu & LE +/-contact & LI impl. & & objet 2D/3D \\
\hline au coin & au N & de $N$ & non & & angle & LE + contact & & contact & rue, cheminée \\
\hline au contact & au N & de $N$ & non & & surface & $\mathrm{LE}+$ contact & & contact & \\
\hline au chevet & au N & de N & oui & & lim. totale & LE +/-contact & & dist. près & HUM (= « lit de HUM ») \\
\hline au détour & au $\mathrm{N}$ & de $\mathrm{N}$ & non & & portion courbe & LI, LE +contact & & & voie \\
\hline au dos & au N & de N & oui & & surface verso & LE +contact & & & feuille, tableau \\
\hline au faîte & au N & de $N$ & non & & portion vertic. supér. & LI, LE +contact & & & bât, végét \\
\hline au fin fond & au fin $\mathrm{N}$ & de $N$ & non & & $\begin{array}{l}\text { lim. opposée à } \\
\text { ouverture }\end{array}$ & LE +/-contact & Ll impl & & territoire \\
\hline au fond & au N & de $N$ & oui & & $\begin{array}{l}\text { lim. opposée à } \\
\text { ouverture }\end{array}$ & LE +/-contact & Ll impl & & 3D ouvert d'un côté \\
\hline au large & au N & de N & oui & & lim. maritime & LE -contact & & & côte, ville côtière \\
\hline au long & au N & de $N$ & non & & lim. longue & LE +/-contact & & & objet allongé \\
\hline au milieu & au N & de $N$ & oui & & milieu & LE + contact & LI impl. & & objet 1D/2D/3D, collection \\
\hline au nez et à la barbe & au $\mathrm{N}$ et à la $\mathrm{N}$ & de N & non & DET poss. & lim. front. antér. & LE -contact & & dist. près & HUM \\
\hline au niveau & au N & de $N$ & non & & lim. totale & LE +/-contact & & alignement & \\
\hline au nord & au N & de $N$ & oui & & portion card. nord & LE +/-contact & & & \\
\hline au nord-est & au N & de $N$ & oui & & portion card. nord-est & LE +/-contact & & & \\
\hline au nord-ouest & au N & de $N$ & oui & & portion card. nord-ouest & LE +/-contact & & & \\
\hline au pied & au N & de $N$ & non & & portion vertic. infér. & LE + contact & & & objet solidaire du sol \\
\hline au plus profond & au plus $\mathrm{N}$ & de N & non & & lim. totale & $\mathrm{LI}$ & LI impl. & & 3D \\
\hline au pourtour & au N & de $N$ & non & & lim. circulaire & LE +/-contact & & & \\
\hline au ras & au N & de $N$ & non & & surface & LE + contact & & alignement & \\
\hline au recto & au N & de $N$ & oui & & surface recto & LE + contact & & & feuille \\
\hline au revers & au N & de $N$ & oui & DET poss. & surface verso & LE +contact & & & \\
\hline au sein & au N & de $N$ & non & & milieu & LE + contact & Ll impl. & & objet 3D ou collection \\
\hline au sommet & au N & de $N$ & oui & DET poss. & portion vertic. supér. & LI, LE +contact & & & solidaire du sol \\
\hline au sud & au N & de N & oui & & portion card. sud & LE +/-contact & & & \\
\hline au sud-est & au N & de $N$ & oui & & portion card. sud-est & LE +/-contact & & & \\
\hline
\end{tabular}




\begin{tabular}{|c|c|c|c|c|c|c|c|c|c|c|}
\hline au sud-ouest & & au N & de N & oui & & portion card. sud-ouest & LE +/-contact & & & \\
\hline au tournant & & au $\mathrm{N}$ & de N & non & & portion courbe & LI, LE + contact & & & voie \\
\hline au travers & & au $\mathrm{N}$ & de $N$ & oui & & lim. latér. : 2 & $\mathrm{LI}$ & holist. & & \\
\hline au verso & & au N & de $N$ & oui & & surface verso & LE + contact & & & feuille \\
\hline au voisinage & & au N & de $\mathrm{N}$ & non & & lim. totale & LE -contact & & dist. près & \\
\hline $\begin{array}{l}\text { au voisinage } \\
\text { immédiat }\end{array}$ & & au N immédiat & de N & non & & lim. totale & LE -contact & & dist. près & \\
\hline au-dedans & au-dedans & au-N & de $N$ & oui & & lim. totale & LI & & & \\
\hline au-dehors & au dehors & au-N & de $N$ & oui & & lim. totale & LE +/-contact & & & \\
\hline au-delà & au-delà & au-N & de N & oui & & lim. front. postér. & LE +/-contact & déict. & & \\
\hline au-dessous & au dessous & au-N & de $N$ & oui & & lim. vertic. infér. & LE +/-contact & & & \\
\hline au-dessus & au dessus & au-N & de $\mathrm{N}$ & \begin{tabular}{|l|} 
oui \\
\end{tabular} & & lim. vertic. supér. & LE +/-contact & & & \\
\hline auprès & & simple & de N & non & & lim. totale & LE -contact & & dist. près & \\
\hline autour & & simple & de $\mathrm{N}$ & oui & & lim. circulaire & LE +/-contact & & & \\
\hline aux abords & & $\operatorname{aux} \mathrm{N}$ & de N & non & & lim. totale & LE -contact & & dist. près & lieu \\
\hline aux alentours & & $\operatorname{aux} \mathrm{N}$ & de $N$ & oui & & lim. circulaire & LE +/-contact & & & \\
\hline aux confins & & $\operatorname{aux} \mathrm{N}$ & de N & non & & lim. totale & LI, LE + contact & & & lieu \\
\hline aux côtés & & $\operatorname{aux} \mathrm{N}$ & de N & non & DET poss. & portion latér. & LE + contact & & & HUM \\
\hline aux environs & & $\operatorname{aux~N}$ & de $\mathrm{N}$ & non & & lim. circulaire & LE +/-contact & & & lieu \\
\hline aux pieds & & $\operatorname{aux} \mathrm{N}$ & de $\mathrm{N}$ & non & DET poss. & portion vertic. infér. & LE + contact & & & HUM \\
\hline aux portes & & $\operatorname{aux} \mathrm{N}$ & de $\mathrm{N}$ & non & & lim. ouverture & LE + contact & & & ville \\
\hline avant & & simple & $\mathrm{N}$ & oui & & lim. front. antér. & LE +/-contact & déict./essent. & & \\
\hline chez & & simple & $\mathrm{N}$ & non & & lim. totale = "domicile" & $\mathrm{LI}$ & & & HUM \\
\hline contre & & simple & $\mathrm{N}$ & non & & lim. vertic. latér. & LE + contact & & & \\
\hline côte à côte & & $\mathrm{NàN}$ & $\begin{array}{l}\text { avec } \mathrm{N} \text {; } \\
\text { récipr. }\end{array}$ & oui & & portion latér. & LE + contact & & & \\
\hline dans & & simple & $\mathrm{N}$ & non & ADV "dedans" & lim. totale & $\mathrm{LI}$ & & & \\
\hline dans les parages & & dans les $\mathrm{N}$ & de $\mathrm{N}$ & oui & DET démonstr & lim. circulaire & LE +/-contact & & & lieu \\
\hline de bas en haut & & de $\mathrm{N}$ en $\mathrm{N}$ & de $\mathrm{N}$ & oui & & portion vertic. : 2 & $\mathrm{LI}$ & holist. & & objet vertical polarisé \\
\hline de ce côté & & de ceN & de N & oui & & portion latér. & LE +/-contact & déict. & & \\
\hline de ce côté-ci & & de ce N-ci & de $\mathrm{N}$ & oui & & portion latér. & LE +/-contact & déict. & & \\
\hline de ce côté-là & & de ce N-là & de N & oui & & portion latér. & LE +/-contact & déict. & & \\
\hline de chaque côté & & de chaque $\mathrm{N}$ & de $\mathrm{N}$ & oui & & portion latér. : 2 & LE +/-contact & & & \\
\hline de haut en bas & & de $\mathrm{N}$ en $\mathrm{N}$ & de N & oui & & portion vertic. : 2 & $\mathrm{LI}$ & holist. & & objet vertical polarisé \\
\hline de l'autre côté & & de l'autre $\mathrm{N}$ & de N & oui & & portion latér. opposée & LE +/-contact & déict. & & \\
\hline de part et d'autre & & de $\mathrm{N}$ et de DET & de $\mathrm{N}$ & oui & & portion latér. : 2 & LE +/-contact & & & \\
\hline
\end{tabular}




\begin{tabular}{|c|c|c|c|c|c|c|c|c|}
\hline derrière & simple & $\mathrm{N}$ & oui & lim. front. postér. & LE +/-contact & déict./essent. & & \\
\hline devant & simple & $\mathrm{N}$ & oui & lim. front. antér. & LE +/-contact & déict./essent. & & \\
\hline dos à dos & $\mathrm{NàN}$ & $\begin{array}{l}\text { avec } \mathrm{N} \text {; } \\
\text { récipr. }\end{array}$ & oui & lim. front. postér. & LE + contact & essent. & dos à dos & HUM \\
\hline du bas en haut & du $\mathrm{N}$ en $\mathrm{N}$ & de N & oui & portion vertic. : 2 & $\mathrm{LI}$ & holist. & & objet vertical polarisé \\
\hline du côté & du N & de $N$ & non & portion latér. & LE -contact & & dist. près & \\
\hline du côté droit & du N ADJ & de $N$ & oui & lim. latér. droite & LI, LE + contact & déict./essent. & & \\
\hline du côté gauche & du N ADJ & de $N$ & oui & lim. latér. gauche & LI, LE + contact & déict./essent. & & \\
\hline du haut en bas & du $\mathrm{N}$ en $\mathrm{N}$ & de N & oui & portion vertic. : 2 & $\mathrm{LI}$ & holist. & & objet vertical polarisé \\
\hline d'un bord à l'autre & d'un N à l'autre & de N & oui & lim. latér. : 2 & $\mathrm{LI}$ & holist. & & \\
\hline d'un bout à l'autre & d'un N à l'autre & de $\mathrm{N}$ & oui & portion extrême : 2 & $\mathrm{LI}$ & holist. & & objet allongé \\
\hline en & simple & $\mathrm{N}$ & non & lim. totale & $\mathrm{LI}$ & & & $\mathrm{N}$ propre toponyme \\
\hline en amont & en $\mathrm{N}$ & de $\mathrm{N}$ & oui & $\begin{array}{l}\text { obj.sur cours d'eau: } \\
\text { supér. }\end{array}$ & LE +/-contact & & & sur un cours d'eau \\
\hline en arrière & en $\mathrm{N}$ & de $N$ & oui & portion front. postér. & LE +/-contact & & & \\
\hline en aval & en $\mathrm{N}$ & de N & oui & $\begin{array}{l}\text { obj.sur cours d'eau: } \\
\text { infér. }\end{array}$ & LE +/-contact & & & sur un cours d'eau \\
\hline en avant & en $\mathrm{N}$ & de N & oui & portion front. antér. & LE +/-contact & & & \\
\hline en bas & en $\mathrm{N}$ & de N & oui & portion vertic. infér. & LI, LE + contact & & & \\
\hline en bordure & en $\mathrm{N}$ & de $N$ & non & lim. latér. & LI, LE + contact & & & $\begin{array}{l}\text { surf. longue, circ./creuse, } \\
\text { eau }\end{array}$ \\
\hline en contrebas & en $\mathrm{N}$ & de N & oui & portion vertic. infér. & LE + contact & & & \\
\hline en deçà & en ADV & de $\mathrm{N}$ & oui & lim. front. antér. & LE +/-contact & déict. & & \\
\hline en dedans & en $\mathrm{N}$ & de N & oui & lim. totale & $\mathrm{LI}$ & & & \\
\hline en dehors & en $\mathrm{N}$ & de $N$ & oui & lim. totale & LE +/-contact & & & \\
\hline en dessous & en $\mathrm{N}$ & de N & oui & lim. vertic. infér. & LE +/-contact & & & \\
\hline en dessus & en $\mathrm{N}$ & de N & oui & lim. vertic. supér. & LE +/-contact & & & \\
\hline en face & en $\mathrm{N}$ & de $\mathrm{N}$ & oui & lim. front. antér. & LE -contact & & & \\
\hline en haut & en $\mathrm{N}$ & de $\mathrm{N}$ & oui & portion vertic. supér. & LI, LE + contact & & & \\
\hline en plein centre & en plein $\mathrm{N}$ & de $N$ & oui & milieu & LE + contact & Ll impl. & & objet 2D/3D \\
\hline en plein dans & en plein PREP & $\mathrm{N}$ & non & lim. totale & $\mathrm{LI}$ & & & \\
\hline en plein milieu & en plein $\mathrm{N}$ & de $N$ & oui & milieu & LE + contact & LI impl. & & objet 1D/2D/3D, collection \\
\hline en plein sur & en plein PREP & $\mathrm{N}$ & non & surface & LE + contact & & & \\
\hline en queue & en $\mathrm{N}$ & de N & oui & portion front. postér. & LI, LE + contact & & & \\
\hline en regard & en $\mathrm{N}$ & de $\mathrm{N}$ & oui & lim. front. antér. & LE -contact & & & \\
\hline en retrait & en $\mathrm{N}$ & de $\mathrm{N}$ & oui & lim. front. antér. & LE -contact & & dist. près & \\
\hline en surplomb & en $\mathrm{N}$ & de N & oui & lim. totale & LE -contact & & d'aplomb & \\
\hline
\end{tabular}




\begin{tabular}{|c|c|c|c|c|c|c|c|c|c|c|}
\hline en tête & & en $\mathrm{N}$ & de N & oui & & portion front. antér. & LI, LE +contact & & & \\
\hline en travers & & en $\mathrm{N}$ & de N & oui & & lim. latér. : 2 & LI & holist. & & objet allongé \\
\hline entre & & simple & $\begin{array}{l}\mathrm{N} \text { et } \mathrm{N} \text {, les } \\
\mathrm{N}\end{array}$ & non & & lim. totale : 2 & LE -contact & & & \\
\hline face & & simple & à N & non & & lim. antér. front. & LE -contact & & & \\
\hline face à face & & $\mathrm{N}$ àN & $\begin{array}{l}\text { avec N ; } \\
\text { récipr. }\end{array}$ & oui & & lim. front. antér. & LE +/-contact & essent. & face à face & \\
\hline hors & & simple & de N & non & ADV "dehors" & lim. totale & LE +/-contact & & & \\
\hline le long & & leN & de N & non & & lim. longue & LE +/-contact & holist. & & objet allongé \\
\hline Ioin & & simple & de $N$ & oui & PRO "en" & lim. totale & LE -contact & & dist. loin & \\
\hline Ioin derrière & & Ioin PREP & $\mathrm{N}$ & oui & & lim. front. postér. & LE +/-contact & & dist. loin & \\
\hline loin devant & & loin PREP & $\mathrm{N}$ & oui & & lim. front. antér. & LE +/-contact & & dist. loin & \\
\hline nez à nez & & $\overline{N a ̀ N}$ & $\begin{array}{l}\text { avec N ; } \\
\text { récipr. }\end{array}$ & oui & & lim. front. antér. & LE -contact & & & HUM \\
\hline par & & simple & Nplur & non & & lim. latér. : 2 & LI & holist. & & \\
\hline par-delà & & par-ADV & $\mathrm{N}$ & non & & lim. front. postér. & LE +/-contact & déict. & & \\
\hline par-derrière & & par-PREP & $\mathrm{N}$ & oui & & lim. front. postér. & LE +/-contact & & & \\
\hline par-dessous & & par-PREP & $\bar{N}$ & oui & & lim. vertic. infér. & LE +/-contact & & & \\
\hline par-dessus & & par-PREP & $\overline{\mathrm{N}}$ & oui & & lim. vertic. supér. & LE +/-contact & & & \\
\hline par-devant & & par-PREP & $\mathrm{N}$ & oui & & lim. front. antér. & LE +/-contact & & & \\
\hline parmi & & simple & $\bar{N}$ & non & & lim. totale plur. & $\mathrm{LI}$ & & & collection \\
\hline près & & simple & de N & oui & PRO "en" & lim. totale & LE -contact & & dist. près & \\
\hline sous & & simple & $\mathrm{N}$ & non & $\begin{array}{l}\text { ADV } \\
\text { "dessous" }\end{array}$ & lim. vertic. infér. & LE +/-contact & & & \\
\hline sous le nez & & sous le $\mathrm{N}$ & $\mathrm{deN}$ & non & DET poss. & lim. front. antér. & LE -contact & & dist. près & HUM \\
\hline sous les yeux & & sous les $\mathrm{N}$ & de N & non & DET poss. & lim. front. antér. & LE -contact & & dist. près & HUM \\
\hline sur \#1 & & simple & $\mathrm{N}$ & non & ADV "dessus" & lim. vertic. supér. & LE +contact & & & \\
\hline sur \#2 & & simple & $\bar{N}$ & non & ADV "dessus" & surface & LE + contact & & & \\
\hline sur \#3 & & simple & $\mathrm{N}$ & non & & lim. vertic. supér. & LE -contact & & & \\
\hline sur la droite & & sur le $\mathrm{N}$ & $\mathrm{deN}$ & oui & DET poss. & lim. latér. droite & LE +/-contact & déict./essent. & & \\
\hline sur la gauche & & sur la $\mathrm{N}$ & de N & oui & DET poss. & lim. latér. gauche & LE +/-contact & déict./essent. & & \\
\hline tout à côté & & tout PREP & $\mathrm{deN}$ & oui & & portion latér. & LE +/-contact & & dist. près & \\
\hline tout à l'entour & tout alentour & tout PREP & $\mathrm{deN}$ & oui & & lim. circulaire & LE +/-contact & holist. & & \\
\hline tout au bout & & tout PREP & $\mathrm{deN}$ & oui & & portion extrême & LI, LE + contact & déict. & & \\
\hline tout au fond & & tout PREP & de N & oui & & $\begin{array}{l}\text { lim. opposée à } \\
\text { ouverture }\end{array}$ & LE +/-contact & Ll impl. & & 3D ouvert d'un côté \\
\hline tout au long & & tout PREP & de N & oui & & lim. longue & LE +/-contact & holist. & & objet allongé \\
\hline tout au milieu & & tout PREP & de N & oui & & milieu & LE +contact & LI impl. & & objet 1D/2D/3D, collection \\
\hline
\end{tabular}




\begin{tabular}{|c|c|c|c|c|c|c|c|c|c|}
\hline tout autour & tout PREP & de N & oui & & lim. circulaire & LE +/-contact & holist. & & \\
\hline tout contre & tout PREP & de N & oui & & lim. vertic. latér. & LE + contact & & & \\
\hline tout du long & tout du N & de N & oui & & lim. longue & LE +/-contact & holist. & & objet allongé \\
\hline tout en bas & tout PREP & de N & oui & & portion vertic. infér. & LI, LE + contact & & & \\
\hline tout en haut & tout PREP & de N & oui & & portion vertic. supér. & LI, LE + contact & & & \\
\hline tout le long & tout PREP & de N & oui & & lim. longue & LE +/-contact & holist. & & objet allongé \\
\hline tout près & tout PREP & de N & oui & PRO "en" & lim. totale & LE -contact & & dist. près & \\
\hline très loin & très PREP & de N & oui & PRO "en" & lim. totale & LE -contact & & dist. loin & \\
\hline très loin derrière & $\begin{array}{l}\text { très PREP } \\
\text { PREP }\end{array}$ & $\mathrm{N}$ & oui & & lim. front. postér. & LE +/-contact & & dist. loin & \\
\hline très loin devant & $\begin{array}{l}\text { très PREP } \\
\text { PREP }\end{array}$ & $\mathrm{N}$ & oui & & lim. front. antér. & LE +/-contact & & dist. loin & \\
\hline très près & très PREP & de N & oui & PRO "en" & lim. totale & LE -contact & & dist. près & \\
\hline vers & simple & $\mathrm{N}$ & non & & lim. totale & LE -contact & & dist. près & \\
\hline vis-à-vis & $\mathrm{NàN}$ & $\begin{array}{l}\text { de N; } \\
\text { récipr. }\end{array}$ & non & & lim. front. antér. & $\mathrm{LE}+/$-contact & & & HUM \\
\hline
\end{tabular}


SHS Web of Conferences 1 (2012)

DOI 10.1051/shsconf/20120100114

(C) aux auteurs, publié par EDP Sciences, 2012 